\title{
Modelling motivational dynamics: demonstrating when, why, and how we self-regulate motivation
}

\author{
Laura B. Thomas \\ Liverpool John Moores University
}

\author{
Joanne Hudson \\ Swansea University
}

Emily J. Oliver

Durham University

\begin{abstract}
Motivation quality affects the initiation and maintenance of behavior and physical and psychological health. Despite this, we understand little about how situational fluctuations occur and are regulated. In this paper we analyze the utility of applying basic psychological needs theory (a sub theory of self-determination theory) and reversal theory as frameworks for understanding motivational dynamics. Specifically, we posit a causal model linking acute consequences of need satisfaction and the purpose and direction of meta-motivational state shifts. This model is tested in two sequential experiments, demonstrating: (i) that thwarting or satisfying psychological needs increases meta-motivational reversal frequency and (ii) that individuals use meta-motivational shifts to compensate for imbalances in need satisfaction. Broad-ranging implications include informing therapeutic support for preventing maladaptive emotions and behaviors and promoting psychological health and well-being. In respect to modelling the dynamics of human motivation, this study adds clarity to understanding when (following need deprivation), why (to regain and balance need satisfaction), and how (through changing metamotivational states) we self-regulate.
\end{abstract}

Keywords: self determination theory; reversal theory; dynamic motivation; balanced need satisfaction; need restoration

\section{Understanding motivational dynamics}

Motivation is a key area of psychological investigation due to the benefits associated with understanding the processes involved in initiating, maintaining, and withdrawing from activities. To date we have a comprehensive understanding of "when" and "why" people are motivated for volitional behavior, including a range of motives (e.g., achievement; Duda \& Nicholls, 1992), goals (e.g., extrinsic rewards or personal development; Elliott \& Dweck, 1988), and need pursuits (e.g., psychological needs: belonging, autonomy and competence; Deci \& Ryan, 1985). However, one aspect of motivation that is poorly understood is the way it changes over time. In particular, few studies have explored how acute motivational changes are perceived, managed, and regulated

Laura B. Thomas, School of Sport and Exercise Sciences, Liverpool John Moores University; Joanne Hudson, Applied Sports, Technology and Exercise Medicine Research Centre, School of Sport and Exercise Sciences, College of Engineering, Swansea University; Emily J. Oliver, School of Applied Social Sciences, Durham University.

Correspondence concerning this article should be addressed to Laura B. Thomas. E-mail: 1.b.thomas@1jmu.ac.uk and the resultant short-term effects on behavior, health, and well-being.

Basic psychological needs theory (BPNT), a sub-theory of self determination theory (SDT; Deci \& Ryan, 1985, 2000), holds considerable appeal when attempting to understand the changeability in human motivation and its relationship with health indices (e.g., psychological well-being). Specifically, SDT makes clear hypotheses of how characteristics of the proximal social environment act as precursors to motivational changes and subsequent alterations in well- and ill-being (see Deci \& Ryan, 2000). It is well evidenced in the literature that environments satisfying the basic psychological needs for autonomy, competence, and relatedness contribute to growth, intrinsic motivation, and indications of wellness (e.g., self esteem and life satisfaction: Deci et al., 2001; well-being: Reis, Sheldon, Gable, Roscoe, \& Ryan, 2000; see Ryan, Huta, \& Deci, 2008). Research has additionally demonstrated that achieving balanced need satisfaction across all three needs is preferable to achieving similar levels of collective, or total, need satisfaction but with greater variability between needs (e.g., Sheldon \& Niemiec, 2006). Together, these bodies of evidence suggest that when environments satisfy needs, and more specifically, satisfy all needs, motivation and well-being are optimized. 
Table 1

Summary of reversal theory states, motives and theorized associations with the basic psychological needs discussed in Self-Determination Theory

\begin{tabular}{lll}
\hline State (Motive) & State Description & Aligned SDT Needs \\
\hline Telic (achievement) & Achievement itself or progression towards & Competence \\
Paratelic (fun) & Partaking in activity for its own sake & Autonomy \\
Mastery (power) & Feeling tough, hardy, and resilient & Competence \\
Sympathy (love) & Feelings of sensitivity, tenderness, and caring & Relatedness \\
Conformist (fitting in) & Fitting in with others; conforming & Relatedness \\
Negativist (freedom) & Breaking free from rules & Autonomy \\
Autic (individuation) & Being free from rules & Autonomy \\
Alloic (transcendence) & Feeling part of and identifying with others & Relatedness \\
\hline
\end{tabular}

The undermining effects of need frustration on motivation and well-being have similarly been well established. Persistent thwarting of the innate psychological needs has been associated with compensatory activity, need substitutes, nonself determined regulatory styles, and rigid behavior patterns (Deci \& Ryan, 2000; Ryan, Deci, Grolnick, \& La Guardia, 2006; Vansteenkiste \& Ryan, 2013). Whilst these coping mechanisms might provide some form of collateral satisfaction, they ultimately detract from well-being (Deci \& Ryan, 2000).

The negative outcomes associated with acute need thwarting are theorized to result in an immediate cost to an individual's psychological health (e.g., negative affect and disengagement). This, however, conflicts with more general motivational literature advocating that deprivation of any fundamental need should lead to a process of restoration (e.g., Fiske, 2004; Hull, 1943; Maslow, 1943; Radel, Pelletier, Sarrazin, \& Milyavskaya, 2011; Veltkamp, Aarts, \& Custers, 2009). For example, in line with Baumeister and Leary's (1995) criteria for identifying a need, we would expect an individual to engage in goal-orientated behavior to satisfy any deprivation. More recently, facilitators of these restoration processes have been identified, with Radel, Pelletier, Sarrazin, and Milyavskaya (2011) demonstrating enhanced accessibility and an approach bias for autonomy following its deprivation. They concluded that experiencing autonomy frustration led to cognitive changes, predisposing individuals to regain the deprived need which might ultimately affect downstream processes that are subject to conscious control (e.g., judgement, opinions, and behavior). Evidence of such a "restorative motive" to replenish the basic psychological needs outlined in SDT was also demonstrated by Sheldon and Gunz (2009). Participants reported an increased desire to satisfy unmet psychological needs allowing for a more balanced satisfaction of the basic needs (Sheldon \& Gunz, 2009; Sheldon \& Niemiec, 2006).

Whilst balanced need satisfaction has important implications for well-being, it is unlikely that many environments allow the basic needs to all be satisfied simultaneously, and to the same degree, thus we are frequently likely to experience an imbalance in need satisfaction. It is expected that to achieve balance individuals turn their attention to less satisfied needs and, to some extent, unmet needs have precedence over met needs (Deci \& Ryan, 2000). However, it is currently not known how we "turn our attention" to unmet needs and how we identify and adjust precedence.

Whilst SDT discourse provides a strong and comprehensive understanding of the environments that support and detract from well-being (the satisfaction and persistent thwarting of basic needs, respectively), the restorative nature and regulation of need pursuits has received limited theoretical and empirical attention/investigation. In the present paper we posit that our understanding of this process might be enhanced through the application of a theory of motivation primarily concerned with motivational dynamics, that is, reversal theory (Apter, 1982).

\section{A model for understanding motivational changes: rever- sal theory}

According to reversal theory (Apter, 1982, 2001), an individual's motivation moves dynamically through four mutually exclusive pairs of meta-motivational states (MMS). Each MMS is characterized by a certain way of interpreting some aspect of one's own motivation (Apter, 1989, 2001; see Table 1) and is associated with a need ${ }^{1}$ or motive that should be satisfied whilst experiencing that state. To be considered "psychologically healthy" people should reverse between states on a regular and frequent basis, thus experiencing a broad range of felt emotions (Apter, 2001). Inhibited reversals are associated with psychological ill health, a restricted range of negative emotions (e.g., anxiety if stuck in the telic state

\footnotetext{
${ }^{1}$ Both theories are concerned with the satisfaction of needs, however, reversal theory's eight needs would be considered by SDT as acquired motives as they are not innate, organismic necessities, required for on-going psychological growth, integrity, and wellbeing (Deci \& Ryan, 2000). For this reason the needs associated with MMS will be termed "motives" throughout this paper.
} 
or depression if stuck in the paratelic state in a low arousal environment; Apter, 1989) and inappropriate states.

Historically the reversal process has been considered to be predominantly reactive, with theorists arguing that individuals cannot consciously, directly, or voluntarily induce a reversal on demand (Apter, 1982). Three reversal-inducing agents are presented in the literature: frustration, when an individual's motives are not satisfied; satiation, postulated to be an entirely internal process with reversals being increasingly likely with the passage of time, and contingent events, that is, a change in surroundings (Apter, 2001). Despite the reversal process being fundamental to the theory, literature surrounding the process lacks depth and clarity. Specifically, it is unclear how and at what level of frustration or satiation a reversal might occur, or whether it is possible to predict the direction and type of reversal an individual is likely to experience. There is also little justification for excluding purposeful as opposed to reactive reversals from the theory, in a way that seems inconsistent with active attempts at coping or optimising well-being that an individual might make. Consideration of purposeful reversals has recently been theorized, for example, Desselles (2013) suggests that states can be called upon through self-conditioning, however the extent to which this is possible requires empirical research (Apter, 2014; Desselles, 2013).

Furthermore, if the purpose of reversals is to experience varied states and in doing so, support health, then is it conceivable that reversals may have a functional role in terms of facilitating restoration of need satisfaction and balance. This proposition is strengthened if the motives made salient within different MMS are seen as ways to facilitate satisfaction of higher order needs as outlined by SDT. For example, autonomy in SDT terms is the degree to which the individual feels volition: the organismic desire to self-organize experience and behavior and to engage in activities in line with one's integrated sense of self (Angyal, 1965; deCharms, 1968; Deci, 1980; Ryan \& Connell, 1989; Sheldon \& Elliot, 1999). Motives from reversal theory that might act to fulfill the need for autonomy include fun whilst in the paratelic state (partaking in the activity for its own sake, similar to intrinsic forms of behavioral regulation), freedom whilst in the negativistic state (breaking free from rules which are considered restricting and controlling, if this is done volitionally and not reactively, ${ }^{2}$ and individuation (being individual, separate and independent) pursued in the autic state. Reversals to these states following deprivation of autonomy, or imbalance caused by high levels of competence or relatedness satisfaction, could therefore be seen as attempts at restoration.

In line with Radel et al.'s (2011) temporal need threat model (based on the General Adaptation Syndrome model; Selye, 1946), we are proposing that individuals might act in an adaptive manner by switching MMS when need thwarting is first experienced. During the initial alarm and response stages of Radel et al.'s model, individuals are expected to allocate resources to fight against thwarting and adapt their functioning. Recognising and changing the priority of a need and reversing to an alternative MMS targeting that need, would be an adaptive restorative process. These adaptive attempts, however, are still recognized to have costs. At the exhaustion stage thwarting has been prolonged and the resources to cope are empty or depleted (Radel et al., 2011). Aligned with SDT discourse, the exhaustion phase is associated with the individual relinquishing the thwarted need, a lack of motivation for the activity, development of need substitutes and compensatory motives, and rigid behavioral patterns. These outcomes are entirely consistent with the proposed consequences of inhibited reversals (e.g., Apter, 1982; Deci \& Ryan, 2000; Radel et al., 2011).

\section{Study 1: Summary and Hypotheses}

The core postulate of this research is that MMS reversals provide a mechanism by which balanced satisfaction of an individual's innate psychological needs is achieved. In essence, we expected that when the social environment was manipulated to undermine need satisfaction and balance, increased restorative efforts would be observed in the form of MMS reversals. As such, in the first exploration of the propositions we hypothesized that conditions that actively thwarted or satisfied specific basic needs would be associated with significantly more reversals than observed in a control condition. To test this hypothesis, we used an established experimental protocol (cf. Eghrari, Deci et al., 1997), randomly allocating participants to environmental conditions designed to thwart or satisfy the basic psychological needs outlined in SDT. We hypothesized that the need-thwarting condition would induce a frustration-based reversal, as thwarting of a need simultaneously prevents the satisfaction of the motives associated with each state and identifies to the individual that a change in motivational focus is required. We also hypothesized that prolonged satisfaction of a specific need would induce a satiation-based reversal, operating to enable a balance of need satisfaction, through reversing from a state associated with a satisfied need to a state associated with an alternative, less satisfied, need.

\section{Study 1 Method}

Participants. Seventy-one participants were recruited to take part in the research as part of an undergraduate course; no credit was received for participation $\left(M_{\text {age }}=20.06\right.$ years,

\footnotetext{
${ }^{2}$ The association between freedom and autonomy has previously been discussed in SDT literature, which states that autonomy concerns the experience of both integration and freedom (Deci \& Ryan, 2000). However, reversal theory's motive for freedom lacks the concordance to self that is encompassed in the SDT conceptualization of autonomy.
} 
Table 2

Stimuli presented during the adapted Stroop Task to assess participants' active MMS

\begin{tabular}{llllllll}
\hline Telic & Paratelic & Conformist & Negativistic & Sympathy & Mastery & Alloic & Autic \\
\hline Goal & Risks & Conform & Defiant & Affectionate & Competition & Altruistic & Individual \\
Serious & Thrills & Obedient & Stubborn & Love & Power & Supporting & Egotistic \\
Future & Playful & Compliant & Rebellious & Sympathetic & Supremacy & Collective & Independence \\
Accomplishment & Spontaneous & Respectful & Innovative & Tenderness & Control & Selfless & Individuality \\
Purpose & Present & Rules & Rebel & Caring & Contest & Empathy & Myself \\
Meaning & Carefree & Cooperation & Provocative & Harmony & Dominance & Altruism & Selfish \\
Cautious & Immediate & Norms & Angry & Kindness & Aggressive & Unity & Self \\
Calm & Humor & Agreeable & Contradict & Sensitivity & Resilience & Give & Ego \\
\hline
\end{tabular}

Note. The adapted Stroop task's development and validation are discussed in Thomas et al. (2015). An average response latency for each state is computed (total of eight response times). Participants' active state is classified as the state with the shortest response latency, in line with the incongruency effect demonstrated in the development of the measure (Thomas et al., 2015).

$\mathrm{SD}=2.15 ; 53$ males, 18 females). Participants were fluent in written and spoken English, which was the first language for 63 of the participants. Following departmental ethical approval, all participants provided informed consent prior to the start of the study.

Measures. The Adapted Stroop Task (Thomas, Hudson, $\&$ Oliver, 2015) was used to assess participants' active MMS. The protocol consisted of 80 stimuli presented in a randomized order in one of four colors: red, green, blue and black (10 stimuli per MMS; see Table 2) taking on average 110s to complete. Participants were instructed to indicate the color of the word as quickly as possible whilst making as few errors as possible. Participants' response time to each stimulus was recorded and average response times per state computed.

Similar to the original Stroop effect and subsequent research regarding emotions (Kunde \& Mauer, 2008; Stroop, 1935) the adapted Stroop has previously demonstrated an incongruency effect (Thomas et al., 2015). That is, greater cognitive effort is required to process incongruent than congruent stimuli; thus, attending to words of opposite valence to the current motivational state exerts greater disruption and interference (Kunde \& Mauer, 2008). The theorized "confusion" or enhanced processing that results from an incongruent stimulus is somewhat consistent with paradigms advocating that threatening stimuli affect attentional disengagement, effectively "capturing" an individual's attention for longer before they can attend to a secondary stimulus (e.g., Fox, Russo, \& Bowles, 2001). If an incongruent stimulus functions as a threat to the status quo, one might expect longer response latencies for these stimuli than for contingent stimuli. Given this, Thomas et al.'s MMS Stroop classifies participants' active states as those with the shortest response latencies. Count data of the rate of change in participants' active state within each MMS pair was calculated allowing each participant's reversal frequency to be computed [(total $n$ reversals/potential pair reversals)*100]. ${ }^{3}$

Manipulation Checks. The Basic Psychological Needs Scale-General (BPNS-G; Deci \& Ryan, 2000) was adapted to measure satisfaction of the basic psychological needs of autonomy, competence, and relatedness during the study. The BPNS includes 21 items: seven relating to autonomy (e.g., "During the study I felt free to express my ideas and opinions"), six relating to competence (e.g., "I felt a sense of accomplishment from completing the study") and eight assessing relatedness (e.g., "I got along with the researcher"). Participants responded to each item on a 1- (Not at all true) to 7-point (Very true) Likert scale; higher scores indicate a higher level of need satisfaction. Gagné (2003) reported coefficient alphas of $.69, .71$ and .86 for the autonomy, competence, and relatedness subscales, respectively.

The Psychological Need Thwarting Scale (PNTS; Bartholomew, Ntoumanis, Ryan, \& Thøgersen-Ntoumani, 2011) was adapted to measure need thwarting during the study. The PNTS consists of 12 items comprising three subscales: autonomy thwarting (e.g., "I felt pushed to behave in certain ways"), competence thwarting (e.g., "During the study I was made to feel incapable"), and relatedness thwarting (e.g., "I felt rejected by the researcher"). Responses for all three sub-scales were provided on a Likert scale ranging from 1 (Not at all true) to 7 (Very true); higher scores indicate a higher level of need thwarting. Each of the need thwarting subscales demonstrated satisfactory internal reliability in the measure's development (autonomy $=.84$; competence $=.88$; relatedness $=.84$; Bartholomew et al., 2011).

Environmental Conditions. Using randomization software, we assigned participants to one of seven environmental conditions: thwarting of autonomy, competence, and relatedness ( $n=12,9$ and 9, respectively), high satisfaction of autonomy, competence, and relatedness $(n=14,5$ and 13,

\footnotetext{
${ }^{3}$ Total $\mathrm{n}$ reversals represents the number of reversals experienced by the participant throughout the 10 cognitive tasks (see Procedure for more details). Potential pair reversals represents the total number of possible reversals that could be assessed during the experimental task (change between 4 pairs of states across 9 time points $=36$ potential reversals).
} 
respectively), or moderate satisfaction $(n=9)$. Participants' data were then collapsed across conditions forming three environmental conditions: high need satisfaction $(n=32)$, need thwarting $(n=30)$ and moderate need satisfaction $(n=9)$. Collapsing participants' data into three conditions allowed the research questions of the effects of need thwarting and need satiation to be tested whilst examining any potential differences between manipulation of the three basic needs. Unequal group sizes were not problematic for the analysis conducted.

Environmental Manipulation. Environmental manipulations followed previously validated protocols (c.f. Deci et al., 1994; Sheldon \& Filak, 2008) in which interactions with the researcher, phrasing of standardized instruction sheets, and performance feedback received were dependent on condition. A detailed description of each condition is provided below (example of materials used to create the environmental conditions can be obtained by contacting the primary researcher).

Autonomy Manipulation. In line with Deci et al. (1994), three contextual factors were manipulated to create the autonomy supportive and thwarting environment: rationale, acknowledgement, and language. In the autonomy supportive condition participants were provided with a rationale for engaging in the study, the primary researcher acknowledged the participant (e.g., recognising that participants might not find the activity interesting or enjoyable), and used language conveying choice (e.g., "might" and "could" as opposed to "have to" and "must"). In contrast, participants in the autonomy deprivation condition were not given a meaningful rationale to engage in the activity, the participant's perspective was not acknowledged during the activity, and autonomy thwarting language was used (e.g., "have" and "must" as opposed to "might" and "could"). In addition, participants were repeatedly reminded of the "rules" regarding engagement in the task, which were displayed visually throughout the testing session.

Competence Manipulation. In line with Sheldon and Filak (2008), competence supporting language was used to create a need-supportive environment (e.g., "Let's see how well you do"). In addition to this, participants were given standardized competence satisfying performance feedback, in the form of verbal and visual feedback after each trial (e.g., "Well done! You are in the top 10\% of participants") expressing high levels of task mastery. In contrast, competence thwarting language was used to create the competence thwarting condition (e.g., "A sense of how poorly you do in the beginning") and standardized competence thwarting performance feedback in the form of verbal and visual feedback (e.g., "Maybe you will do better next time as currently, you are in the bottom $10 \%$ of participants") expressing low levels of task mastery.

Relatedness Manipulation. In line with Sheldon and Filak (2008), relatedness supporting statements such as "I care about your learning style" and "I have confidence in you" were presented to participants, in both verbal and written instructional sets, prior to and post task engagement. The primary researcher took time to get to know the participant prior to participation, offered refreshments, and the opportunity to have breaks throughout the testing session. In contrast, in the relatedness thwarting condition the primary researcher used relatedness thwarting statements such as "I am only concerned with your performance in the task, please keep your opinions to yourself." The primary researcher appeared disinterested in the participant, used an incorrect name to address them, appeared busy with other tasks, and left the participant waiting with no instruction.

Moderate Need Satisfaction Manipulation. Participants' needs were partially satisfied, however this was not to the same extent as the forced satiation condition. Participants were informed of the task to be completed and their progress throughout the session (e.g., "You have completed 5 puzzles, you are half way through"). Participants received standardized visual and verbal feedback informing them of a consistently average level of performance (e.g., "You are in the top 60\%").

\section{Procedure}

Participants read a standardized instruction sheet corresponding with their environmental condition and had the opportunity to ask questions. An element of deception was used, with participants believing the purpose of the research was to enhance understanding of motivation and concentration assessed through completing an automated computer package. The software consisted of ten cognitive tasks (five boggle puzzles and five Sudoku grids), each two minutes in duration, displayed in a randomized order. However, the true purpose of the tasks was to provide opportunity to implement need manipulation techniques, creating the experimental environmental conditions (e.g., standardized performance feedback, in line with environmental condition, on completion of each cognitive task to manipulate competence thwarting or satisfaction). After each feedback point, participants completed the adapted Stroop task. After ten tasks participants completed the BPNS-G and PNTS before being thanked and debriefed.

\section{Data Analysis}

One-way ANOVAs were performed to assess the effectiveness of the environmental manipulation, with Bonferroni post-hoc tests used to determine differences in perceived need satisfaction and thwarting between conditions.

Multilevel modeling was used to examine group and intrapersonal changes in participants' MMS. Multilevel techniques were employed to overcome the errors associated with data nested within environmental conditions and over time. 
Such dependencies are associated with compromised standard methods of statistical analysis, resulting in underestimation of the standard error, thus increasing the likelihood of a false significant result (Hox, 2010). To analyze between group differences, a two level model, in which individual participants are level one units (i) and environmental conditions are level two units (j), was applied.

\section{Study 1 Results}

Manipulation Check. One way ANOVAs revealed a significant difference in total need satisfaction and need thwarting between the high satisfaction, need thwarting and moderate satisfaction conditions $(F(2,64)=16.74, p<.001$ and $F(2,68)=5.87, p=.004$, respectively). Bonferroni follow up tests revealed differences in the expected direction. Participants in the high satisfaction $(M=116.65, \mathrm{SD}$ $=14.56 ; g=1.44,95 \%$ CI $[0.88,2.00])$ and moderate satisfaction conditions $(M=116.14, \mathrm{SD}=16.32 ; g=1.40,95 \%$ CI $[0.60,2.21])$ were significantly more satisfied than participants in the thwarting condition $(M=96.55, \mathrm{SD}=12.84)$. Additionally, participants in the need thwarting condition $(M=25.23, \mathrm{SD}=9.94 ; g=.83,95 \%$ CI $[0.32,1.35]) \mathrm{re}-$ ported feeling significantly more thwarted than participants in the high satisfaction condition $(M=18.09, \mathrm{SD}=6.75)$ but not the moderate satisfaction condition. Taken together, the results support the effectiveness of the environmental manipulations.

Hypothesis Testing. Hypothesis: Conditions that actively thwart or satisfy one or more of SDT's basic needs will induce a meta-motivational state reversal, with significantly more reversals observed under these conditions than a moderate need satisfaction condition.

To allow for dependency in reversal frequency within environmental conditions and to examine the extent of between environmental variation in reversal frequency the following multilevel model was run:

$$
y_{i j}=\beta 0+\mu_{j}+\epsilon_{i j}
$$

where $y_{i j}$ is reversal frequency [(total $n$ reversals/potential pair reversals) $* 100$ ] of participant $i$ in condition $j, \beta 0$ is the overall mean across environmental conditions, $\mu_{j}$ is the effect of condition $j$ on the dependent variable, and $\epsilon_{i j}$ is a participant level residual. The condition effects, $\mu_{j}$, are assumed to follow a normal distribution with mean zero and variance $\sigma_{\mu}^{2}$.

The overall mean reversal frequency (across conditions) was estimated as 48.58 . The between condition (level 2) variance in reversal frequency was estimated as $\sigma_{\mu}^{2}=6.62$, and the within environmental condition (level 1) variance was estimated as $\sigma_{\epsilon}^{2}=60.58$. Thus, the total variance was 67.20. A variance partition (ICC) of .09 indicating that $10 \%$ of the variance in reversal frequency can be attributed to differences between environmental conditions reinforced the need to continue to model the hierarchical data structure.

To examine the difference in mean number of reversals between the environmental conditions the following model was run with the natural satisfaction condition acting as the reference category.

$$
\begin{gathered}
y_{i j}=\text { Moderate Satisfaction }+ \text { High Satisfaction }+ \text { Need } \\
\text { Thwarting }+\epsilon_{i j} \\
y_{i j}=42.560(2.560)+8.166(2.868)+7.772(2.902)+\epsilon_{i j} \\
\epsilon_{i j} \sim N\left(0 ; \sigma_{\epsilon}^{2}\right) \quad \sigma_{\epsilon}^{2}=57.372(9.839)
\end{gathered}
$$

A one way ANOVA revealed a significant difference in the number of reversals between the environmental conditions $(F(2,65)=249.72, p=.020$; observed power .753); participants in the high need satisfaction and thwarting conditions experienced significantly more reversals than participants in the moderate satisfaction condition $(g=-2.87,95 \%$ CI $[-3.82,-1.89] ; g=-2.69,95 \%$ CI $[-3.64,-1.73]$, respectively). Thus, the hypothesis was accepted.

\section{Study 1 Discussion}

Contrary to SDT theorizing regarding negative responses to need thwarting, the preliminary findings support the adaptive responses of the alarm and response stage outlined by Radel et al.'s (2011) model. In line with our expectations, environments that undermined need satisfaction and balance were associated with restorative efforts in the form of increased MMS reversals. The increased reversal frequency associated with the need deprivation and imbalanced conditions provides some initial support for theoretical congruence with SDT in terms of mechanisms underpinning the "when" and "how" of the reversal process. Specifically, it is thought that need thwarting underpins frustration-based reversals as the active thwarting of a need prevents the satisfaction of the motives associated with the experienced MMS and identifies that a change in motivational focus is required. An imbalance in need satisfaction, caused by high levels of need satisfaction at the expense of others, is thought to underpin satiation-based reversals. The diametrically opposed MMS pairs compliment the balance of needs as discussed in SDT; needs cannot all be satisfied at one time; however, needs can be satisfied over time and this could be considered a feasible reason for reversals, enabling individuals to attempt to optimize psychological satisfaction (Apter, 2001).

In line with Apter's proposition, the results support the conceptualisation of satiation reversals that operate not merely due to the passage of time, but due to over-exposure to a given aspect of environmental conditions, irrespective of whether this aspect is associated with positive outcomes or not. This is the first time that this element of reversal theory discourse (termed "plentitude" by Apter, 2013) has been demonstrated. 


\section{Study 2 Introduction}

Although Study 1 provides preliminary support for a need restorative function of MMS reversals, evidenced through increased reversal frequency in response to need deprivation/imbalance, the nature of the reversal remains unclear. Central to this theorisation is the proposition that MMS motives feed into the higher order needs outlined in SDT and that MMS reversals are somewhat purposeful. Of particular interest to establish are: (i) whether the change in MMS is to prioritize a deprived need, thus orientating towards balance and (ii) whether the change in MMS was effective at influencing subsequent levels of need satisfaction.

As outlined in brief above, the motives of freedom, fun, and individuation associated with the negativistic, paratelic, and autic states might act to fulfil the need for autonomy. Similarities between MMS motives and the fundamental needs for competence and relatedness are also evident. Competence in SDT concerns the degree to which individuals feel effective in their interactions with the environment and experience opportunities in which to express their capabilities (Ryan \& Deci, 2002). Apter (2001) describes achievement itself, or progress towards achievement, as a motive when in a telic state. The connection between competence and achievement has been highlighted previously, stating that the achievement motive is, to a substantial degree, based on the innate need for competence (Koestner \& McClelland, 1990), but also encompasses behaviors or ideations based in ego involvement or approval motives and is therefore not truly innate in SDT terms.

Relatedness is the desire to feel connected to others, to love and care and to be loved and cared for (Baumeister \& Leary, 1995; Bowlby, 1958; Harlow, 1958; Ryan, 1993). We propose motives from reversal theory aligned with satisfaction of the need for relatedness include fitting in whilst in the conformist state (if by fitting in this means feeling close/connected to others), transcendence whilst in the alloic state, feeling part of, and identifying with others, and love whilst in the sympathy state, described as feelings of sensitivity, tenderness and caring, which would typify meaningful interpersonal connections associated with relatedness.

The motive for power whilst in the mastery state is more difficult to clearly link to higher order needs outlined in SDT. Power in reversal theory is described as the need to feel tough, hardy and resilient (Apter, 2001), therefore does not directly relate to any SDT needs, nor does it appear to correspond well with SDT's conceptualization of power as an extrinsic motive or compensatory reaction to need thwarting. With hardiness and resilience defined as a capability for enduring difficult conditions and recovering quickly from setbacks (e.g., Collins, 1995) an argument can be made that such capabilities perhaps reflect a robust or durable sense of competence, hence, pursuit of reversal theory's "power" motive might function to satisfy competence needs.
The ability to reverse between MMS to prioritize a deprived need would require a structured and strategic approach by the individual, considering task selection, optimal duration of engagement in any given activity or context, and monitoring of alternatives as well as future events. To this end, individuals might plan around upcoming events, potentially prioritizing needs that have been, or they anticipate will be, deprived by cultivating need satisfying experiences. Previous research has provided some initial evidence suggesting that the restorative motive, orientating towards balanced need satisfaction, involves cognitive changes predisposing individuals to regain the deprived need which ultimately affected downstream processes that are subject to conscious control (e.g., judgement, opinions, and behavior; Radel et al., 2011). Applying concepts from reversal theory we similarly argue that restorative motives might be achieved through acute cognitive changes; however, the structured nature of reversal theory provides a more purposeful framework by which restoration might be achieved. Specifically, the recognition of a need to reverse in order to help cultivate activities to satisfy prioritized needs, might prompt a purposeful reversal.

This proposal is antagonistic to reversal theory discourse which considers the reversal process to be subconscious, and that individuals cannot consciously, directly, or voluntarily induce a reversal on demand (Apter, 1982). However, in line with more recent developments in reversal theory, it is recognized that MMS reversals can be induced indirectly through manipulation of the three reversal inducing mechanisms: waiting for satiation to occur, deliberate use of frustration, and contingent events (e.g., a deliberate change in the environment; see Desselles \& Apter, 2013 for a full discussion). Historically, contingent events have been described as a change in situation or physical environment that trigger a reversal, for example, experiencing the effect of a drug, tripping during an enjoyable mountain climb, or entering a church. However, Apter (2013) highlights that contingent events should include more than the externally observable changes in the environment. In line with the phenomenological nature of reversal theory, the "situation" should consider how the situation is perceived by the individual, for example, changes in memories, imagination, and what the person attends to. As such, it is argued that the recognition of a need to cultivate a climate or activity to satisfy an unmet need might act as a contingent event, inducing a MMS reversal. A conscious decision to change focus, and attend to something new, could therefore induce a reversal to a state congruent with satisfying the prioritized need.

The aim of this second exploratory study was to extend the findings of Study 1 by examining if participants purposefully used MMS reversals to compensate for decrements or imbalances in need satisfaction. Specifically, our hypothesis was: 
1. Following a period of need deprivation, individuals will reverse to, or maintain, a MMS that is congruent with satisfying the prioritized need:

a. Reversals to paratelic, negativistic and autic states will be most evident under conditions of autonomy deprivation;

b. Reversals to conformist, alloic, and sympathy states will be most evident under conditions of relatedness deprivation;

c. Reversals to telic and mastery states will be most evident under conditions of competence deprivation.

2. After experiencing, and prior to experiencing further, need deprivation, threatened needs will take priority; individuals will actively cultivate a MMS through which to experience targeted need satisfaction, thus protecting long-term balanced need satisfaction.

\section{Study 2 Method}

Participants. Eighty participants were recruited from undergraduate psychology module practical activity $(n=72)$ and an opportunistic sample $(n=8)$; no course credit was received for participation $\left(M_{\text {age }}=22.04\right.$ years, $\mathrm{SD}=7.24 ; 53$ males, 27 females). ${ }^{4}$ As with Study 1, the sample is reflective of a UK university population and the local population in terms of ethnic diversity, however this is not diverse relative to the general population. Participants were fluent in written and spoken English, which was the first language for 73 of the participants. Following departmental ethical approval, all participants provided informed consent prior to the commencement of the study.

Measures. We calculated the average response latencies to autonomy, competence, and relatedness-congruent MMS, with prioritized need satisfaction inferred by shorter response latency, using the Adapted Stroop Task (Thomas et al., 2015, see Study 1). The Stroop task was completed twice during the free choice period: first at the start, assessing active state in the initial stage of satisfying prioritized needs, and second at the end, assessing active state in the final stages of satisfying prioritized needs, before experiencing further anticipated need deprivation. Participants' response time to each stimulus presented in the adapted Stroop protocol was recorded, and average response times per state computed. The average response latency to autonomy, competence, and relatednesscongruent MMS was calculated (e.g., autonomy response latency $=[$ Paratelic latency + Negativistic latency + Autic latency]/3) with prioritized need satisfaction demonstrated by smaller response latency in line with the incongruency effect.

The Balanced Measure of Psychological Needs-General (BMPN-G; Sheldon \& Hilpert, 2012) was completed on three occasions: at baseline, on task completion, after a free choice period. The 18-item BMPN-G assesses both satisfaction and dissatisfaction of the three basic psychological needs outlined in SDT, resulting in three items per sub-scale. A final aggregate score was calculated by subtracting need dissatisfaction from need satisfaction. Balanced need satisfaction was calculated as the sum of absolute differences between the three need aggregate satisfaction scores (Balance= $[\mathrm{A}-\mathrm{C}]+[\mathrm{A}-\mathrm{R}]+[\mathrm{C}-\mathrm{R}])$. Each item was rated on a 1- (Not at all true) to 5-point (Very true) Likert scale.

Environmental Conditions. Three experimental conditions were produced: autonomy, competence, and relatedness deprivation. Each condition was designed to create imbalanced need satisfaction: ample opportunity to satisfy two of the basic psychological needs, but limited opportunity to satisfy the remaining need. The environmental manipulation of need satisfaction followed the manipulation techniques used in Study 1 and previously validated protocols for need satisfaction and need thwarting (c.f. Deci et al., 1994; Sheldon \& Filak, 2008).

Procedure. Participants were randomly assigned to one of three need deprivation conditions using randomization software: autonomy $(n=31)$, competence $(n=36)$ and relatedness $(n=13)$. Mid data collection manipulation check revealed that relatedness thwarting techniques were unsuccessful, as such, this need deprivation condition was excluded from further randomization and data analyses. After condition allocation, participants read a standardized instruction sheet corresponding to their environmental condition. They were informed that the testing session would consist of three stages: two experimental trials (one of which was fictitious) separated by a break. Participants were not aware that the purpose of the experimental trial was to create a period of need imbalance, and the purpose of the free choice period was to provide participants with an opportunity to satisfy any deprived needs.

Experimental Trial. Participants attempted to complete as many puzzles as possible within the 15-minute trial, before completing the BMPN. Throughout the experimental trial the primary researcher manipulated the environment in line with techniques detailed previously.

Free Choice Period. Participants received a fifteenminute free choice period (see Ryan \& Deci, 2000) which was framed as a mid-task break. The free choice period allowed participants the opportunity to "top up" the deprived need after experiencing one bout of need deprivation, and whilst anticipating further deprivation. During the fifteenminute free choice period participants were informed that they could act volitionally and were then left alone whilst being covertly recorded. Participants' active MMS was assessed during the first five minutes and on completion of the

\footnotetext{
${ }^{4} \mathrm{Mid}$ data collection power analysis revealed that in order for an effect of this size to be detected ( $80 \%$ chance) as significant at the $5 \%$ level, a total sample size of 80 participants would be required.
} 
Table 3

Results from repeated measures ANOVAs assessing differences in satisfaction and dissatisfaction of the psychological needs

\begin{tabular}{|c|c|c|c|c|}
\hline Sub-scale & df & $\mathrm{F}$ & $\mathrm{p}$ & $\eta_{\rho}^{2}$ \\
\hline \multicolumn{5}{|c|}{ Autonomy Deprivation Condition } \\
\hline Satisfaction & 2,58 & 12.13 & $<.001$ & 0.422 \\
\hline Dissatisfaction & 2,58 & 35.23 & $<.001$ & 0.690 \\
\hline \multicolumn{5}{|c|}{ Competence Deprivation Condition } \\
\hline Satisfaction & 2,70 & 35.73 & $<.001$ & 0.661 \\
\hline Dissatisfaction & 2,70 & 80.26 & $<.001$ & 0.838 \\
\hline \multicolumn{5}{|c|}{ Relatedness Deprivation Condition } \\
\hline Satisfaction & $1.40,24$ & 1.9 & 0.171 & 0.137 \\
\hline Dissatisfaction & 2,24 & 5.6 & 0.010 & 0.318 \\
\hline
\end{tabular}

free choice period. On completion of the testing session participants completed the post task measures, were thanked, and debriefed.

\section{Study 2 Results}

Randomisation Check - Balanced Measure of Psychological Needs-General. To assess the level of need satisfaction provided in participants' day-to-day lives prior to attending the testing session, a one-way ANOVA was conducted. Results demonstrated nonsignificant differences between groups for general need satisfaction, dissatisfaction, and aggregate satisfaction across the three psychological needs $\left(p=.135-.587 ; \eta_{\rho}^{2}=.013-0.51\right)$ suggesting that participants' level of need satisfaction prior to attending the session was similar.

Manipulation Check - Balanced Measure of Psychological Needs-Experimental Trial. To assess the overall effectiveness of the environmental manipulation, differences in satisfaction and dissatisfaction of the three psychological needs during the experimental trial (BMPN-ET) were assessed using repeated measures ANOVAs. Bonferroni posthoc analyses were performed to determine differences in need satisfaction during the experimental trial. The results are discussed below with a summary provided in Table 3.

The results suggest that the environmental manipulation was successful for the competence deprivation condition and partially successful for the autonomy deprivation condition. Contrary to expectations, participants in the autonomy deprivation condition experienced similar levels of autonomy and competence dissatisfaction, however this was not considered overly problematic due to the higher levels of competence satisfaction experienced. When assessing aggregate need satisfaction, which accounts for both satisfaction and dissatisfaction, in line with expectations, participants experienced lower autonomy aggregate satisfaction $(M=.53$, SD $=4.10)$ than competence aggregate satisfaction $(M=1.93$,
$\mathrm{SD}=3.92 ; p=.058, g=-.355,95 \%$ CI $[-.85,0.16]) ;$ this trend approached significance. Analysis of the BMPN-ET suggests that environmental manipulation for the relatedness deprivation condition was not successful; satisfaction and dissatisfaction of the three needs were similar across environmental conditions. As such, participants in the relatedness condition were excluded from further analysis.

\section{Hypothesis Testing}

Hypothesis 1: following a period of need deprivation individuals will reverse to, or maintain, a meta-motivational state congruent with satisfying the prioritized need.

Repeated measures ANOVAs examining differences in response to need congruent state latencies in the initial stage of satisfying prioritized needs (first Stroop task), and assessing participants' active state in the final stages of satisfying prioritized needs (second Stroop task) were conducted. The first Stroop task revealed a nonsignificant main effect of need latencies $\left(F(2,26)=1.043 ; p=.355, \eta_{\rho}^{2}=\right.$ $.016)$ and condition $\mathrm{x}$ need latency interaction $(F(2,126)=$ 2.552; $\left.p=.082, \eta_{\rho}^{2}=.039\right)$. The second Stroop task revealed a nonsignificant main effect of need latencies $(F(2,126)=$ $.773 ; p=.464, .012)$ but a significant condition $\mathrm{x}$ need latency interaction $\left(F(2,126)=3.414 ; p=.036, \eta_{\rho}^{2}=.051\right)$. However, Bonferroni follow up tests revealed nonsignificant differences.

The results provide no support for the hypothesis; after a period of need deprivation participants' active metamotivational state was not congruent with those proposed to satisfy the deprived need.

Hypothesis 2: after experiencing need deprivation, and anticipating further need deprivation, threatened needs will take priority; individuals will actively cultivate activities to enable need satisfaction, thus protecting long term balanced need satisfaction.

Experience of Need Satisfaction. To assess if participants' experience of need satisfaction changed during the free choice period, a repeated measures ANOVA was conducted examining need satisfaction in general (BMPN-G), during the experimental trials (BMPN-ET), and after the free choice period (BMPN-C) for each condition. Results demonstrated a significant effect of time for both the autonomy deprivation $\left(F(1.585,45.969)=35.035 ; p<.001 ; \eta_{\rho}^{2}=.547\right)$ and competence deprivation conditions $(F(1.621,56.725)=$ 86.314; $\left.p<.001 ; \eta_{\rho}^{2}=.711\right)$.

Bonferroni post-hoc analysis revealed that participants in the autonomy deprivation condition reported significantly greater autonomy satisfaction at baseline $(M=12.20, \mathrm{SD}$ $=7.70)$ and after the free choice period $(M=10.10$, SD $=2.28)$ than during the experimental trial $(M=7.70, \mathrm{SD}=$ 3.13; $p<.001, g=0.76,95 \%$ CI $[0.24,1.27] ; p=.001, g=$ $0.87,95 \%$ CI $[-1.39,-0.34]$, respectively). A significant difference in autonomy satisfaction at baseline and after the 
Table 4

Summary of means and standard errors from post-hoc analysis

\begin{tabular}{|c|c|c|c|c|c|}
\hline Sub-scale & Mean Difference & SE & $\mathrm{g}$ & \multicolumn{2}{|c|}{$95 \% \mathrm{CI}$} \\
\hline \multicolumn{6}{|l|}{ Autonomy Deprivation Condition } \\
\hline \multicolumn{6}{|l|}{ Need Satisfaction } \\
\hline Autonomy - Competence & $-1.90 * *$ & 2.65 & -0.70 & -1.21 & -0.88 \\
\hline Autonomy - Relatedness & $-\mathbf{2 . 7 0} * * *$ & 2.31 & -1.09 & -1.62 & -0.56 \\
\hline Competence - Relatedness & -0.80 & 1.82 & -0.43 & -0.93 & 0.08 \\
\hline \multicolumn{6}{|l|}{ Need Deprivation } \\
\hline Autonomy - Competence & -0.50 & 2.42 & -0.20 & -0.70 & 0.29 \\
\hline Autonomy - Relatedness & 3.00*** & 1.83 & 1.57 & 1.00 & 2.14 \\
\hline Competence - Relatedness & $3.50 * * *$ & 1.94 & 1.72 & 1.14 & 2.30 \\
\hline \multicolumn{6}{|l|}{ Competence Deprivation Condition } \\
\hline \multicolumn{6}{|l|}{ Need Satisfaction } \\
\hline Competence - Autonomy & $-3.98 * * *$ & 2.50 & -1.57 & -2.10 & 1.04 \\
\hline Competence - Relatedness & $-3.92 * * *$ & 2.23 & -1.75 & -2.29 & -1.21 \\
\hline Autonomy - Relatedness & 0.06 & 2.49 & 0.02 & -0.44 & 0.49 \\
\hline \multicolumn{6}{|l|}{ Need Deprivation } \\
\hline Competence - Autonomy & $2.56 * * *$ & 2.09 & 1.21 & 0.70 & 1.71 \\
\hline Competence - Relatedness & 4.05*** & 1.70 & 2.53 & 1.91 & 3.15 \\
\hline Autonomy - Relatedness & $1.94 *$ & 1.63 & 1.15 & 0.65 & 1.64 \\
\hline \multicolumn{6}{|l|}{ Relatedness Deprivation Condition } \\
\hline \multicolumn{6}{|l|}{ Need Satisfaction } \\
\hline Relatedness - Autonomy & -1.31 & 2.52 & -0.50 & -1.29 & 0.28 \\
\hline Relatedness - Competence & 0.54 & 2.90 & 0.19 & -0.58 & -0.96 \\
\hline Autonomy - Competence & 1.85 & 2.84 & 0.63 & -0.16 & 1.42 \\
\hline \multicolumn{6}{|l|}{ Need Deprivation } \\
\hline Relatedness - Autonomy & -1.00 & 1.38 & -0.78 & -1.57 & 0.02 \\
\hline Relatedness - Competence & $-2.23 *$ & 2.20 & -0.89 & -1.69 & -0.08 \\
\hline Autonomy - Competence & -1.23 & 2.30 & -0.48 & -1.26 & 0.30 \\
\hline
\end{tabular}

Note. The bolded mean differences were predicted to be significantly different. The mean differences were expected to be negative when assessing need satisfaction data (satisfaction of the deprived need is less) and positive when assessing need deprivation data (deprivation of the deprived need is greater).

Table 5

Results from repeated measure ANOVAs examining response latencies (ms) to need congruent states

\begin{tabular}{lccc}
\hline Condition & Autonomy Latency & Competence Latency & Relatedness Latency \\
\hline First Stroop Task & & & \\
$\quad$ Autonomy Deprivation Condition & $\mathbf{6 6 1 . 5 5}(83.87)$ & $666.52(78.19)$ & $665.40(85.31)$ \\
$\quad$ Competence Deprivation Condition & $664.02(94.00)$ & $\mathbf{6 4 9 . 2 2}(89.18)$ & $662.79(91.57)$ \\
Second Stroop Task & & & \\
$\quad$ Autonomy Deprivation Condition & $\mathbf{5 9 9 . 3 6}(60.19)$ & $610.23(72.40)$ & $594.79(61.40)$ \\
Competence Deprivation Condition & $579.46(82.69)$ & $\mathbf{5 8 1 . 7 5}(79.11)$ & $596.44(69.52)$ \\
\hline
\end{tabular}

Note. Within each row the bolded mean is predicted to be smaller than the other means within that row.

free choice period was also evident $(p<.001, g=0.37$, $95 \%$ CI $[-0.14,0.87])$. Significant differences in competence satisfaction were evident between baseline $(M=11.61, \mathrm{SD}$ $=1.48)$ and both during the experimental trial $(M=6.69, \mathrm{SD}$ $=2.23 ; p<.000, g=2.57,95 \% \mathrm{CI}[1.95,3.20])$ and after the free choice period $(M=8.97, \mathrm{SD}=2.06 ; p<.001, g=1.46$, 95\% CI $[0.94,1.98])$, and between the experimental trial and after the free choice period $(p<.001, g=-1.05,95 \%$ CI $[-1.54,-0.56])$. Regardless of environmental condition need satisfaction was greatest pre-trial and significantly higher after the free choice period than during the experimental trial, suggesting partial success at need restoration.

Achieving Balanced Need Satisfaction. A mixed measures ANOVA was used to assess if the experiences of the free choice period allow participants to regain balanced need satisfaction. Results revealed a significant main effect for time $\left(F(2,128)=15.321 ; p<.001 ; \eta_{\rho}^{2}=.193\right.$; observed power .999) and a nonsignificant time $\mathrm{x}$ condition interac- 
tion $\left(F(2,128)=.324 ; p=.724 ; \eta_{\rho}^{2}=.005 ;\right.$ observed power .101). Bonferroni post-hoc analysis revealed significantly greater imbalance after completing the experimental condition $(M=12.64, \mathrm{SD}=8.33)$ than after the free choice pe$\operatorname{riod}(M=9.48, \mathrm{SD}=5.67 ; p=.022, g=.44,95 \% \mathrm{CI}$ $[0.10,0.78])$ regardless of condition.

Results provide support for the hypothesis; irrespective of environmental condition, participants reduced the magnitude of need imbalance created during the experimental trial, through the activities cultivated during the free choice period. As such hypothesis one is partially accepted.

\section{Study 2 Discussion}

Study 2 provides mixed support for the proposition that participants would purposefully use MMS reversals to compensate for decrements and imbalance in need satisfaction. In line with expectations, results demonstrate that during the free choice period, participants successfully reduced the magnitude of need imbalance created during the experimental trial. As such, participants evidenced need restoration. However, it is unclear how the return in balanced need satisfaction occurred, given null findings with respect to hypothesized MMS orientations post-deprivation. It is possible that the increased reversal frequency associated with need thwarting allows for psychodiversity, thus promoting well-being (Apter, 2007). As such, assessing active MMS at two time points during the break period is a limitation of the study; it is possible that many states might have been experienced during this period, but were not all captured. In addition, it is possible that balance automatically re-establishes itself, suggesting that any effects of need thwarting are short lived. Whilst the cause of regained balance is unclear, the finding provides support for the evolutionary perspective of the basic psychological needs within SDT (Deci \& Ryan, 1985) and is consistent with Sheldon and Gunz's (2009) initial research examining the desire to acquire missing experiences. Regardless of environmental condition, need satisfaction was significantly higher following the free choice period than during the experimental trial, suggesting attempts at need restoration.

Taken together there is a body of evidence suggesting that the basic psychological needs within SDT may act as internal motives that direct behavior towards satisfying a need that is not available in the current environment. Achieving balanced need satisfaction allows the individual to reduce the stress and conflict associated with an inappropriate allocation of resources (Sheldon \& Gunz, 2009) and, we posit, experience a broad range of motives and resulting emotions which are associated with optimal psychological health and well-being (Apter, 1982; Apter \& Carter, 2002). As such, individuals who, consciously or unconsciously, assess current need satisfaction levels and adapt accordingly will be at an advantage to those with similar overall need satisfaction but with greater variability.

\section{General Discussion}

The purpose of this multi-study research was to enhance our understanding of how motivation fluctuates over time. In particular, we examined how these fluctuations are experienced and regulated, and the resultant short-term effects on need satisfaction as a conduit to psychological health and well-being. Based on the findings from these two sequential studies, we propose employing principles from SDT and reversal theory in an integrated model that conceptualizes both upstream and downstream processes of motivational shifts (the propositions of which are illustrated in Figure 1). These results provide initial support for the dynamic model, specifically i) the level of need satisfaction experienced acts as a reversal inducing mechanism and ii) following a period of need imbalance people are able to return to a state of more balanced need satisfaction.

In addition to identifying a potential framework by which acute fluctuations in motivation are regulated, the present research has contributed a number of theoretical developments. Study 1 provides the first independent support of the alarm and response stage of Radel and colleagues' (2011) temporal need threat model. Results demonstrate a potential restorative function of MMS reversals, evidenced through increased frequency in response to deprivation. Furthermore, the propositions have been examined in a context where participants are unable to move away from the thwarting, and importantly a more detailed, structured mechanism for the restorative attempt is provided through MMS changes.

Across the two studies there is also evidence supporting two concepts within reversal theory that until now, had not received empirical investigation. Study 1 provides support for a new form of reversal that is induced by the amount of satisfaction experienced and not solely due to the passage of time, termed plentitude (Apter, 2013).The second study tentatively supports the use of indirect reversals; the recognition of a need to prioritize deprived needs acts as a contingent event inducing a change in MMS (Apter, 2013).

The use of indirect reversals was central to the thesis that deprivation of a basic psychological need would induce a reversal to, or maintain, a MMS congruent with satisfying the prioritized need. However, the results are inconclusive. It is possible that the Stroop task was unable to accurately assess active state (e.g., completing the Stroop task induced a MMS reversal; see Thomas et al., 2015), or the deprivation was not severe, prolonged or personal enough to warrant need prioritization (e.g., the level of perceived need thwarting impacted students' behavior and emotions; Hein, Koka, \& Hagger, 2015). As such, the mechanism by which need satisfaction is recouped following a period of deprivation warrants further investigation. 


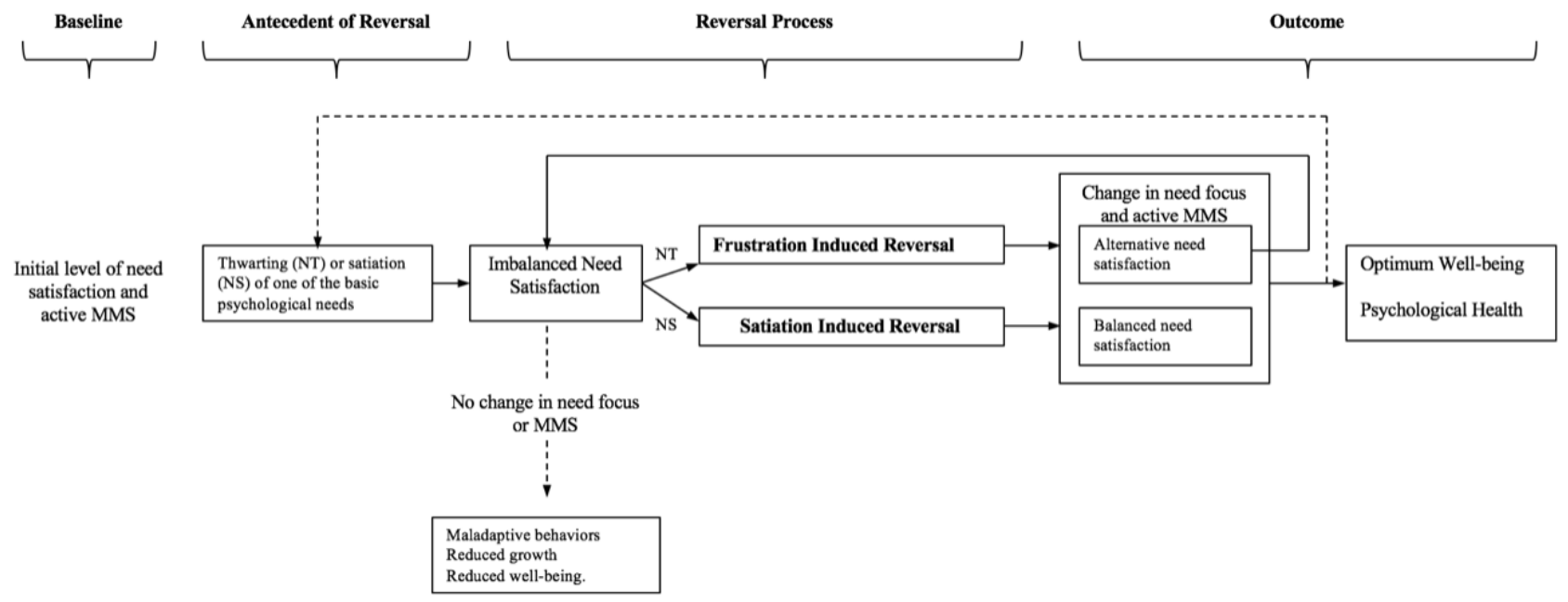

Figure 1. Model displaying the dynamic interplay between SDT and reversal theory. The model illustrates the proposed framework of antecedents of meta-motivational state changes, and the reversal mechanism by which individuals might achieve balanced need satisfaction

The present research raises a number of interesting future research directions. These include considering whether chronic deprivation of a basic psychological need is associated with less adaptive responses in the form of devaluation of the thwarted need. Baumeister (2015) theorizes that wanting something and not getting it will weaken the desire response, evidenced through reduced intensity and frequency of the desire. However, it remains unclear what duration and/or intensity of thwarting will be experienced prior to devaluation occurring, and if/how this devaluation can be reversed.

In addition to an individual's ability to regulate need satisfaction and respond to chronic deprivation, future research should consider the potential moderating role of individual differences. As previously discussed by Sheldon and Gunz (2009) individual differences might moderate the "needs as motives" effect in their ability to recognize and reduce deficits (e.g., an extroverted individual may be more equipped to make new acquaintances than an introverted individual). We argue that individual differences might also moderate the extent of deprivation experienced prior to "accepting defeat." Despite SDT's proposition that the three needs are universal, and so do not vary across people (Deci \& Ryan, 2000), it is possible that individual differences might affect the recognition of deprived needs, desire to attain need satisfying experiences, degree of satisfaction sustained from the activity, and the amount of thwarting experienced prior to need devaluation.

Finally, future research should examine if the restorative effect demonstrated in Study 2 would also be apparent in a sample of participants who are experiencing illbeing/reduced psychological health (e.g., depression, social anxiety, and eating disorders). Whilst the healthy population tolerated acute deprivation/imbalance and returned to baseline levels of satisfaction after a short free choice period, individuals who are experiencing reduced psychological health might be more likely to ruminate on the perceived thwarting/deprivation (e.g., Response Styles Theory; NolenHoeksema, 1991). They might also tolerate lower levels of need deprivation prior to need devaluation, and be less likely to seek activities to promote need satisfaction.

It is worth noting several limitations of the present research. The population used in the two experimental studies is liable to limitations in diversity and size. The samples were restricted in their use of a primarily undergraduate student sample with limited ethnic and racial diversity. Manipulation of participants' basic psychological needs was not always successful (e.g., relatedness in Study 2), and might not have been severe enough to fully test our arguments (as suggested by relatively small changes in the magnitude of satisfaction and thwarting reported by participants). This could be attributable to, and highlights the difficulty in, manipulating the level of satisfaction, particularly when manipulating multiple needs within the same environment. The research does not assess intrapersonal events during the free choice period (e.g., thoughts, feelings, and memories) which have been shown to affect an individual's need satisfaction, emotional regulation, and well-being (Phillippe, Koestner, Beaulieu-Pelletier, Lecours, \& Lekes, 2011). It is possible that intrapersonal changes during this time reflect prioritizing of deprived needs and help to satisfy needs. Finally, assessment of active MMS is challenging as at any given time there can be internal processes or environmental changes that induce reversals (Desselles \& Apter, 2013; Thomas et al., 
2015). The Stroop task is one of two measures available to assess active MMS (cf. Desselles, Murphy, \& Theys, 2014; Reversal Theory State Measure) both of which require ongoing validation though future research.

Despite these limitations, a number of strengths are evident. The results provide evidence supporting autonomy deprivation as a motive for need satisfaction, and so extend the work of Sheldon and Gunz (2009) who found no support for this, attributed to problems in their manipulation. To our knowledge this is the first study to simultaneously manipulate the three basic psychological needs to create an imbalanced environment in an experimental setting. Manipulation checks support the techniques used for two of the three environments. Finally, we have attempted to harmonize contributions from comparable, comprehensive theories in an attempt to achieve a more unified theory, capable of explaining changes in motivation across many domains of behavior (Donovan, 2001; Jesus \& Lens, 2005; Lock \& Latham, 2004; Steel \& König, 2006; Weiner, 1996).

From an applied perspective, the ability of individuals to induce reversals and achieve a balance of need satisfaction might prevent maladaptive behaviors associated with exposure to need thwarting conditions. This has applications in a variety of settings, for example, embedding into counseling services aimed at supporting coping and/or preventing rigid behavior (e.g., cognitive behavioral therapy to treat eating disorders). An individual's ability to correct acute imbalances in need satisfaction suggests that short term deficits might not be detrimental to well-being and growth providing the opportunity to correct imbalance is available in the near future. This might have implications for structuring school days, training courses (e.g., Soldier Initial Training) or work days. The addition of a free time period might impact not only on well-being, but other markers of enjoyment such as adherence and effort.

In sum, the present research enhances our understanding of reversal theory, self determination theory, and more broadly of psychological need satisfaction and motivation. Evidence suggests that prioritizing basic needs might be achieved through purposeful reversals, which contribute to well-being through enabling a balance in need satisfaction (Sheldon \& Niemiec, 2006) and a diverse emotional experience (Apter, 1982). In respect to modelling the dynamics of human motivation, this study adds clarity to understanding when (following need deprivation), why (to regain and balance need satisfaction), and how (through changing metamotivational states) we self-regulate.

\section{References}

Angyal, A. (1965). Neurosis and treatment: A holistic theory. New York: Wiley.

Apter, M. J. (1982). The experience of motivation: The theory of psychological reversals. London: Academic Press.
Apter, M. J. (1989). Reversal theory: Motivation, emotion and personality. London: Routledge.

Apter, M. J. (2001). Motivational styles in everyday life: A guide to reversal theory. Washington, D.C: American Psychological Association.

Apter, M. J. (2013). Developing reversal theory: Some suggestions for future research. Journal of Motivation, Emotion, and Personality, 1, 1-8.

Apter, M. J. (2014). Towards a theory of things: Reversal theory and design. Journal of Motivation, Emotion, and Personality, 2, 3-11.

Apter, M. J., \& Carter, S. (2002). Mentoring and motivational versatility: An exploration of reversal theory. $\mathrm{Ca}$ reer Development International, 7(5), 292- 295.

Bartholomew, K. J., Ntoumanis, N., Ryan, R. M., \& Thøgersen-Ntoumani, C. (2011). Psychological need thwarting in the sport context: Assessing the darker side of athletic experience. Journal of Sport and Exercise Psychology, 33, 75-102.

Baumeister, R. F., \& Leary, M. R. (1995). The need to belong: Desire for interpersonal attachments as a fundamental human motive. Psychological Bulletin, 99(3), 265-272.

Bowlby, J. (1958). The nature of the child's ties to his mother. Journal of Psychoanalysis, 39, 350.

Collins. (1995). Concise dictionary. Glasgow: Harper Collins.

deCharms, R. (1968). Personal causation. New York: Academic.

Deci, E. L. (1980). The psychology of self-determination. Lexington, MA: Heath.

Deci, E. L., Eghrari, H., Patrick, B. C., \& Leone, D. R. (1994). Facilitating internalization: The selfdetermination theory perspective. Journal of Personality, 62(1), 119-142.

Deci, E. L., Huta, V., \& Deci, E. L. (2008). Living well: A self-determination theory perspective on Eudaimonia. Journal of Happiness Studies, 9, 139-170.

Deci, E. L., \& Ryan, R. M. (1985). The general causality orientation scale: Self determination in personality. Journal of Research in Personality, 19, 109-134.

Deci, E. L., \& Ryan, R. M. (2000). The "what" and "why" of goal pursuits: Human needs and the self determination of behaviour. Psychological Inquiry, 11(4), 227-268.

Deci, E. L., Ryan, R. M., Gagnè, M., Leone, D. R., Usunov, J., \& Kornazheva, B. P. (2001). Need satisfaction, motivation, and well-being in the work organizations of a former Eastern Bloc country: A cross-cultural study of selfdetermination. Personality and Social Psychology Bulletin, 27, 930-942.

Desselles, M. L., \& Apter, M. J. (2013). Manipulating motivational states: A review. Journal of Motivation, Emotion, and Personality, 1, 44-49. 
Desselles, M. L., Murphy, S.L., \& Theys, E. R. (2014). The development of the Reversal Theory State Measure. Journal of Motivation, Emotion, and Personality, 2, 10-21.

Donovan, J. J. (2001). Work motivation. In Anderson, N., Ones, D.S., Sinangil, H.K., \& Viswesvaran, C. (Eds.), Handbook of industrial and organisational psychology (pp. 53-77). Thousand Oaks, CA: Sage.

Duda, J. L., \& Nicholls, J. G. (1992). Dimensions of achievement motivation in schoolwork and sport. Journal of Educational Psychology, 84(3), 290-299.

Elliott, E. S., \& Dweck, C. S. (1988). Goals: An approach to motivation and achievement. Journal of Personality and Social Psychology, 54, 5-12.

Fiske, S. T. (2004). Social beings: A core motives approach to social psychology. New York: Wiley.

Fredrickson, B. L. (1998). What good are positive emotions? Review of General Psychology, 2(3), 300-319.

Gagnè, M. (2003). The role of autonomy support and autonomy orientation in prosocial behaviour engagement. $\mathrm{Mo}$ tivation and Emotion, 27(3), 199-223.

Harlow, H. (1958). The nature of love. American Psychologist, 13, 673-685.

Hein, V., Koka, A., \& Hagger, M. S. (2015). Relationships between perceived teachers' controlling behaviour, psychological need thwarting, anger and bullying behaviour in high-school students. Journal of Adolescence, 42, 103 114.

Henry, B., Moffitt, T. E., Caspi, A., Langley, J., \& Silva, P. A. (1994). On the "remembrance of things past": A longitudinal evaluation of the retrospective method. Psychological Assessment, 6(2), 92-101.

Hull, C. (1943). Principles of behaviour. New York: Appleton-Century-Crofts.

Jesus, S. N., \& Lens, W. (2005). An integrated model for the study of teacher motivation. Applied Psychology: An International Review, 54(1), 119-134.

Koestner, R., \& McClelland, D. C. (1990). Perspectives on competence motivation. In L. A. Pervin (Ed.), Handbook of personality: Theory and research (pp. 527-548). Guilford Press.

Locke, E. A., \& Latham, G. P. (2004). What should we do about motivation theory? Six recommendations for the twenty first century. Academy of Management Review, 29(3), 388-403.

Maslow, A. H. (1943). A theory of human motivation. Psychological Review, 50(4), 370-396.

Nolen-Hoeksema, S. (1991). Responses to depression and their effects on the duration of depressive episodes. Journal of Abnormal Psychology, 100, 569-582.

Phillippe, F. L., Koestner, R., Beaulieu-Pelletier, G., Lecours, S., \& Lekes, N. (2011). The role of episodic memories in current and future well-being. Personality and Social Psychology Bulletin, 38(4), 505-519.
Radel, R., Pelletier, L. G., Sarrazin, P., \& Milyavskaya, M. (2011). Restoration process of the need for autonomy: The early alarm stage. Journal of Personality and Social Psychology, 101, 919-934.

Reis, H. T., Sheldon, K. M., Gable, S. L., Roscoe, J., \& Ryan, R. M. (2000). Daily well-being: The role of autonomy, competence, and relatedness. Personality and Social Psychology Bulletin, 26(4), 419-435.

Ryan, R. M. (1993). Agency and organization: Intrinsic motivation, autonomy and the self in psychological development. Lincoln: University of Nebraska Press.

Ryan, R. M., \& Connell, J. P. (1989). Perceived locus of causality and internalization: Examining reasons for acting in two domains. Journal of Personality and Social Psychology, 57(5), 749-761.

Ryan, R. M., \& Deci, E. L. (2000). Intrinsic and extrinsic motivations: Classic definitions and new directions. Contemporary Educational Psychology, 25, 54-67.

Ryan, R. M., \& Deci, E. L. (2002). Overview of selfdetermination theory: An organismic dialectical perspective. In E. L. Deci \& R. M. Ryan (Eds.), Handbook of self determination research (pp. 3-33). New Jersey: John Wiley Sons.

Ryan, R. M., Deci, E. L., Grolnick, W. S., \& La Guardia, J. G. (2006). Autonomy, relatedness, and the self: Their relation to development and psychopathology. In Cicchetti, D. \& Cohen, D.J. (Eds.), Developmental psychopathology: Vol. 1. Theory and methods (pp. 618-655). New York: Wiley \& Sons.

Selye H. (1946). The general adaptation syndrome and the diseases of adaptation. Journal of Clinical Endocrinology 6, 117-231.

Sheldon, K. M., \& Elliot, A. J. (1999). Goal striving, need satisfaction, and longitudinal well-being: The self concordance model. Journal of Personality and Social Psychology, 76(3), 482-497.

Sheldon, K. M., \& Filak, V. (2008). Manipulating autonomy, competence and relatedness support in a game-learning context: New evidence that all three needs matter. British Journal of Social Psychology, 47, 267-283.

Sheldon, K. M., \& Gunz, A. (2009). Psychological needs as basic motives, not just experimental requirements. Journal of Personality, 77(5), 1467-1492.

Sheldon, K. M., \& Hilpert, J. C. (2012). The balanced measure of psychological needs (BMPN) scale: An alternative domain general measure of need satisfaction. Motivation and Emotion, 36, 439-451.

Sheldon, K. M., \& Niemiec, C. P. (2006). It's not the amount that counts: Balanced need satisfaction also affects well being. Journal of Personality and Social Psychology, 91(2), 331-341.

Steel, P., \& König, C. J. (2006). Integrating theories of motivation. Academy of Management Journal, 31(4), 889-913. 
Thomas, L. B., Hudson, J., \& Oliver, E. J. (2015). Development and validation of an implicit measure of metamotivational states. Journal of Motivation, Emotion, and Personality, 4, 15-25.

Vansteenkiste, M., \& Tyan, R. M. (2013). On psychological growth and vulnerability: Basic psychological need satisfaction and need frustration as a unifying principle. Journal of Psychotherapy Integration, 23, 263-280.
Veltkamp, M., Aarts, H., \& Custers, R. (2009). Unravelling the motivational yarn: A framework for understanding the instigation of implicitly motivated behaviour resulting from deprivation and positive affect. European Review of Social Psychology, 20, 345-381.

Weiner, B. (1996). Searching for order in social motivation. Psychological Inquiry, 7(3), 199-216. 